\title{
Association between exposure to fine particulate matter and osteoporosis: a population-based cohort study
}

\author{
G. Adami ${ }^{1}$ (D) G. Cattani $^{2} \cdot$ M. Rossini ${ }^{1} \cdot$ O. Viapiana $^{1} \cdot$ P. Olivi $^{3} \cdot$ G. Orsolini $^{1} \cdot$ E. Bertoldo ${ }^{1} \cdot$ E. Fracassi $^{1} \cdot$ D. Gatti $^{1} \cdot$ \\ A. Fassio ${ }^{1}$
}

Received: 12 April 2021 / Accepted: 30 June 2021 / Published online: 15 July 2021

(C) The Author(s) 2021

\begin{abstract}
Summary Long-term environmental air pollution exposure was associated with osteoporosis' risk in a cohort of women at high risk of fracture. Cortical sites seemed to be more susceptible to the exposure's effect.

Introduction Environmental air pollution has been associated with disruption of bone health at a molecular level. Particulate matter (PM) exposure can simultaneously stimulate bone resorption and halt bone formation. The primary aim of the present study is to describe the association between long-term exposure to PM and osteoporosis in a large cohort of women at high risk of fracture.

Methods Clinical, demographic, and densitometric data were extracted from the DeFRAcalc79 dataset, which gathers data on women at risk for osteoporosis. Data on the monitoring of PM10 and PM2.5 concentrations were retrieved from the Italian institute of environment protection and research (Istituto Superiore per la Protezione e la Ricerca Ambientale, ISPRA). Generalized linear models with robust estimators were employed to determine the relationship between BMD and PM longterm exposure.

Results A total 59,950 women from 110 Italian provinces were included in the study. PM 2.5 exposure was negatively associated with T-score levels at the femoral neck $(\beta-0.005,95 \mathrm{CI}-0.007$ to -0.003$)$ and lumbar spine $(\beta-0.003,95 \% \mathrm{CI}-0.006$ to -0.001). Chronic exposure to PM2.5 above $25 \mu \mathrm{g} / \mathrm{m}^{3}$ was associated with a $16 \%$ higher risk of having osteoporotic T-score at any site (aOR 1.161, 95\% CI 1.105 to 1.220 ), and exposure to PM10 above $30 \mu \mathrm{g} / \mathrm{m}^{3}$ was associated with a $15 \%$ higher risk of having osteoporotic T-score at any site (aOR 1.148, 95\% CI 1.098 to 1.200 ).

Conclusion Long-term exposure to air pollution was associated with higher risk of osteoporosis. Femoral neck site seemed to be more susceptible to the detrimental effect of PM exposure than lumbar spine site.

Key message Exposure to air pollution is associated with osteoporosis, mainly at femoral site.
\end{abstract}

Keywords Bone mineral density $\cdot$ Osteoporosis $\cdot$ Particulate matter $\cdot$ Pollution

G. Adami

giovanni.adami@univr.it

G. Cattani

giorgio.cattani@isprambiente.it

M. Rossini

maurizio.rossini@univr.it

O. Viapiana

ombretta.viapiana@univr.it

P. Olivi

pietro.olivi@aovr.veneto.it

G. Orsolini

giovanniorsolini@gmail.com
E. Bertoldo

eugenia.bertoldo@gmail.com

E. Fracassi

elena.fracassi@univr.it

D. Gatti

davide.gatti@univr.it

A. Fassio

angelo.fassio@yahoo.it

1 Rheumatology Unit, University of Verona, Pz Scuro 10, 37134 Verona, Italy

2 Italian Institute for Environmental Protection and Research, Rome, Italy

3 Orthopedic Unit, University of Verona, Verona, Italy 


\section{Introduction}

Osteoporosis is a chronic disease characterized by low bone mineral density (BMD) and increased risk of fragility fractures [1]. Osteoporosis affects approximately one-third of the women aged 50 years or more, causing a major societal and economic cost [1]. The worldwide burden of osteoporosis is expected to surge in magnitude in the next decades as the populations are rapidly aging [2]. The single, most important, determinant of bone strength and, consequently, fractures risk is BMD. BMD itself is influenced by a number of factors, such as age, body mass index (BMI), menopause, family history of fragility fractures, alcohol intake, smoking status, bone disrupting comorbidities, and intake of some medications. For example, glucocorticoids (GCs) directly affect BMD by inducing osteoblasts and osteocytes apoptosis [3]. Again, inflammatory rheumatic diseases have been associated with higher risk of osteoporosis $[4,5]$. Nevertheless, albeit the knowledge around the determinants of low BMD has constantly expanded in the last decades, there still are unknown aspects. Environmental air pollution is among the novel risk factors that has emerged only in the very last few years as a possible contributor to low BMD; this evidence was particularly pronounced in highly industrialized and polluted areas [6]. The biological rationale underpinning such correlation is strong. Indeed, exposure to fine particulate matter (PM) of diameter of less than $10 \mu \mathrm{m}$ or of less than $2.5 \mu \mathrm{m}$ favors the secretion of receptor activator of nuclear factor-kappa ligand 1 (RANKL), altering the RANKL to osteoprotegerin (OPG) ratio [7]. In addition, exposure to pollutants has been linked with a low-grade inflammation with the release of proinflammatory cytokines, similarly has seen in inflammatory arthritides $[8,9]$. Finally, high concentrations of PM in the lower atmosphere can decrease the amount of UVB that reach the Earth's surface, with, consequently, impaired vitamin D production [10]. However, even if the rationale is strong, air pollution has been neglected as a risk factor for osteoporosis and has not been included in the commonly available fracture risk tools. The primary objective of the present study is to determine and describe, using a web-based fracture risk assessment tool, the association between long-term exposure to fine PM and osteoporosis in a large population-based cohort of women at high risk of fracture.

\section{Material and methods}

We extracted clinical and densitometric data from the DeFRAcalc79 dataset, which was generated using the webbased fracture risk assessment tool, DeFRAcalc79 (https:// defraosteoporosi.it). The DeFRAcalc79 gather data of women at risk of fracture all over Italy collected from June 2016 to January 2020 by 3,326 physicians (both family care practitioners and bone specialists). DeFRAcalc79 considers the following variables for risk calculation: age; weight; height; number and site of prior fragility fractures; parental history of hip and clinical vertebral fractures; GC intake ( $\geq 5 \mathrm{mg} /$ day prednisone equivalent); treatment with adjuvant hormone therapy for breast or prostate cancer; and a diagnosis of rheumatoid arthritis (RA), systemic lupus erythematosus (SLE), psoriatic arthritis (PsA), systemic sclerosis (SS), other connective tissue diseases (CTDs), inflammatory bowel diseases (IBD), chronic obstructive pulmonary diseases (COPD), diabetes, neurological diseases (ND; including Parkinson's disease, multiple sclerosis, and severe physical disability), lumbar spine, and femoral neck T-scores (calculated from the BMD reference range of young same sex individuals). No patients were taking anti-osteoporotic pharmacological treatment at the time of enrollment to the database. The cohort characteristics have been descripted in detail elsewhere $[11,12]$.

We retrieved data on the monitoring of PM10 and PM2.5 concentrations from the Italian institute of environment protection and research (Istituto Superiore per la Protezione e la Ricerca Ambientale, ISPRA) which gather data from air quality stations across the country. The long-term average PM concentrations were the exposure of interest. Every study subject was linked to a PM exposure value, which resulted from the average concentration of urban, rural, and near-traffic stations of the subject province of residency from January 2013 to December 2019.

Group comparisons were performed using a Student's $t$-test and Mann-Whitney U test (for normally and non-normally distributed continuous variables, respectively). Associations between continuous variables were tested using Pearson correlation coefficients. Generalized linear models with robust estimators were employed to identify determinants of osteoporosis (defined as T-score at any site $<-2.5$ ) and relationship between BMD and PM long-term exposure. Exposure to PM was analyzed either as a continuous variable or as a binary variable (exposure thresholds were $30 \mu \mathrm{g} / \mathrm{m}^{3}$ and $25 \mu \mathrm{g} / \mathrm{m}^{3}$ for PM10 and PM2.5, respectively). We sequentially adjusted for confounders. Model 1 included age, body mass index (BMI), presence of prevalent fragility fractures, family history of vertebral or hip fractures, and menopause. Model 2 was further adjusted for glucocorticoid treatment and comorbidities. Model 3 (main model) was further adjusted for the macro-area of residency (stratified as a categorical variable: northern Italy, central Italy, and southern Italy). Differences were considered significant at $p<0.05$. All statistical analyses were performed using SPSS Version 26 (SPSS, Inc., Chicago, IL, USA). Data were anonymized in full compliance with the Italian code of protection of personal data (Legislative Decree 196/03, http://www.camera.it/parlam/leggi/deleghe/03196dl. $\mathrm{htm})$. No identifiers related to patients were provided to the researchers. Results derived from all analyses were produced 
as aggregated summaries, which are not possible to assign, either directly or indirectly, to the individual patients. Informed consent was not required using encrypted retrospective information. This study was approved by the University of Verona ethic committee (prot.1876).

\section{Results}

We collected data from 59,950 women (mean age 65.1 years); most of the population had osteoporotic T-scores (64.5\%), and comorbidities that affect bone health were present in about $15 \%$ of the cohort. Table 1 shows the descriptive characteristics of the study population. A total of 41,219 (68.8\%) subjects were resident in northern Italy, 7,109 (11.9\%) in central Italy, and 11,622 (19.4\%) in southern Italy. We obtained air quality data from 617 air quality stations across 110 Italian provinces. Figure Fig. 1 shows the PM10 2013-2019 average concentrations across Italy. Average exposure to air pollution in the 2013-2019 period in Italy was $16.0 \mu \mathrm{g} / \mathrm{m}^{3}$ as regards PM2.5 and $25.0 \mu \mathrm{g} / \mathrm{m}^{3}$ as regards PM10. Exposure to PM was higher in the Po Valley and in other flat, industrialized and densely populated provinces across Italy.

Table 1 Descriptive characteristics of the cohort

\begin{tabular}{ll}
\hline Overall cohort & $n=59,950$ \\
\hline Age in years ( $\pm \mathrm{SD}, \mathrm{IQR})$ & $65.1( \pm 11.0,58-73)$ \\
Weight in $\mathrm{kg}( \pm \mathrm{SD}, \mathrm{IQR})$ & $62.2( \pm 12.1,54-69)$ \\
Height in cm ( $\pm \mathrm{SD}, \mathrm{IQR})$ & $160.3( \pm 7.6,155-165)$ \\
BMI in kg/m ${ }^{2}( \pm \mathrm{SD}, \mathrm{IQR})$ & $24.2( \pm 4.7,21.2-26.6)$ \\
Femoral neck T-score $( \pm \mathrm{SD}, \mathrm{IQR})$ & $-2.16( \pm 0.94,-2.8$ to -1.6$)$ \\
Lumbar spine T-score $( \pm \mathrm{SD}, \mathrm{IQR})$ & $-2.50( \pm 1.16,-3.21$ to -1.90$)$ \\
T-score <-2.5 at any site $(\%)$ & $38,670(64.5 \%)$ \\
$\%$ 10-year risk of fracture $( \pm \mathrm{SD}, \mathrm{IQR})$ & $19.5( \pm 18.7,7.9-22.9)$ \\
Parental history of fragility fractures $(\%)$ & $14,939(24.9 \%)$ \\
Prevalent fragility fracture $(\%)$ & $20,487(34.2 \%)$ \\
Comorbidities (\%) & \\
None & $46,404(77.4 \%)$ \\
Diabetes & $3,114(5.2 \%)$ \\
Rheumatoid arthritis & $3,008(5.0 \%)$ \\
Psoriatic arthritis & $703(1.2 \%)$ \\
Systemic lupus erythematosus & $294(0.5 \%)$ \\
Systemic sclerosis & $277(0.5 \%)$ \\
Other connective tissue diseases & $1,910(3.2 \%)$ \\
Inflammatory bowel diseases & $942(1.6 \%)$ \\
Chronic obstructive pulmonary disease & $1,614(2.7 \%)$ \\
Parkinson's disease & $412(0.7 \%)$ \\
HIV infection & $235(0.4 \%)$ \\
Multiple sclerosis & $243(0.4 \%)$ \\
Severe physical handicap & $749(1.3 \%)$ \\
\hline &
\end{tabular}

We found a negative association between long-term exposure to PM and T-scores; the point estimates were larger for PM2.5 compared to PM10 and for femoral neck T-scores compared to lumbar spine T-scores. In the unadjusted analyses, we found an association between PM long-term exposure and T-score levels $(p-0.31$ and $p-0.14, p<0.001$ for PM10 and femoral neck and lumbar spine T-scores, respectively, and $p-0.56$ and $p-0.30, p<0.001$ for PM2.5 and femoral neck and lumbar spine T-scores, respectively). The results of the generalized linear models are presented in Table 2. In the main model, PM 2.5 exposure was negatively associated with Tscore levels at the femoral neck (mean difference $-0.015 \mathrm{~T}$ score points per $3 \mu \mathrm{g} / \mathrm{m}^{3}$ increase in PM2.5, 95\% CI -0.021 to -0.009 ). Fitting of the main model was good (deviance/df 0.784). We then considered long-term exposure to PM as a categorical variable (thresholds for average exposure to PM2.5: $25 \mu \mathrm{g} / \mathrm{m}^{3}$ and average exposure to PM10: $30 \mu \mathrm{g} /$ $\mathrm{m}^{3}$ ). We found that being chronically exposed to high levels of PM was associated to an increased risk of osteoporosis (Figure Fig. 2). In the fully adjusted model, subjects chronically exposed to average levels of PM10 above $30 \mu \mathrm{g} / \mathrm{m}^{3}$ had a 15\% higher risk of having osteoporotic T-score at any site (aOR 1.148, 95\% CI 1.098 to 1.200 ), and patients exposed to average concentrations of PM2.5 above $25 \mu \mathrm{g} / \mathrm{m}^{3}$ had a $16 \%$ higher risk of having osteoporotic $\mathrm{T}$-score at any site (aOR 1.161, 95\% CI 1.105 to 1.220). Figures Fig. 3 and Fig. 4 show the risk of osteoporosis stratified by site (femoral neck and lumbar spine).

\section{Discussion}

We conducted an observational, population-based cohort study on the association between long-term exposure to PM and BMD. Overall, we found that exposure to air pollution was negatively associated with BMD levels. In particular, cortical sites (i.e., femoral neck) were more susceptible as compared to trabecular sites (i.e., lumbar spine) to the detrimental effect imposed by PM exposure. PM2.5 was more strongly associated to low BMD compared to PM10.

There is a strong biological rationale supporting our results. Air pollution has been shown to induce oxidative stress not only in the airways but also in bone cells [13]. Indeed, pollutants can directly diminish Wnt signaling activity in the skeleton with detrimental effects on bone formation [14]. Furthermore, exposure to fine PM has been associated with increased serum levels of bone resorption markers [15]. In summary, exposure to air pollutants exerts a dual negative effect on both bone resorption and bone formation; hence it is not surprising that long-term exposure to high levels of air pollutants (i.e., $30 \mu \mathrm{g} / \mathrm{m}^{3}$ of PM10 and $25 \mu \mathrm{g} / \mathrm{m}^{3}$ of PM2.5) was associated with a $15 \%$ higher risk of having osteoporosis. 


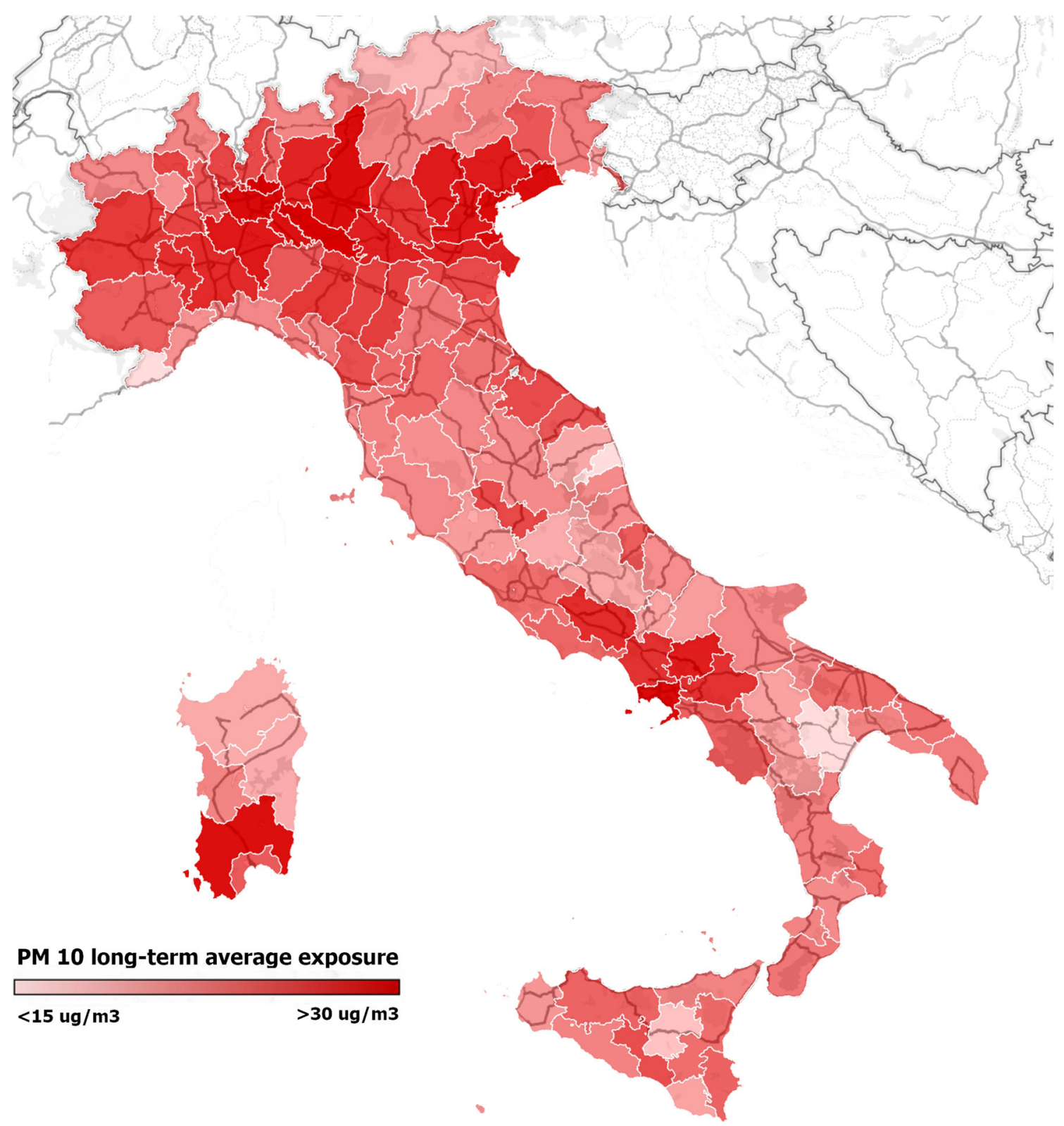

Fig. 1 Long-term exposure to particulate matter (PM) of less than $10 \mu \mathrm{m}$ in Italy (2013-2019 average concentration $\mu \mathrm{g} / \mathrm{m}^{3}$ )

From the generalized linear models, we found a negative association between PM2.5 exposure and BMD in both lumbar spine and femoral neck sites with point estimates larger for femoral neck site. Indeed, we found a peculiar vulnerability of the cortical site to the detrimental effect of air pollution. Interestingly, low-grade inflammation of chronic inflammatory arthritides has been shown to induce cortical bone loss through a RANKL-mediated mechanism $[5,16]$, which is remarkably similar to what occurs after the exposure to environmental air pollution [7]. Indeed, toxic components of fossil combustion can directly increase the secretion of RANKL through the induction of pro-inflammatory cytokines $[7,8]$. The "low-grade inflammation" hypothesis is also supported by the established association between air pollution exposure and systemic markers of inflammation $[17,18]$. However, the aforementioned association between air pollution exposure and low-grade inflammation might be less relevant in older individuals, whom are commonly less exposed to air pollution than younger individuals. In addition, aging is itself associated with a low-grade inflammation, which contributes to the pathogenesis of age-related diseases, such as osteoporosis [19].

Air pollution has been previously linked to low BMD. Several studies had been conducted on this topic, and they all led to similar conclusions. A recent paper by Ranzani and colleagues elegantly demonstrated that both ambient and household air pollution were associated with lower bone mineral content (BMC) [20]. The magnitude of the effect on bone health demonstrated by the authors was comparable to our 
Table 2 Association between long-term exposure to particulate matter (PM) and T-score

\begin{tabular}{|c|c|c|c|c|}
\hline Site, model & PM10 $-\beta$ and $95 \%$ CI & $p$ value & PM2.5 - $\beta$ and $95 \%$ CI & $p$ value \\
\hline \multicolumn{5}{|l|}{ Femoral neck } \\
\hline Unadjusted & $-0.005(-0.007$ to -0.004$)$ & $<0.0001$ & $-0.010(-0.012$ to -0.009$)$ & $<0.0001$ \\
\hline Model 1 & $-0.003(-0.004$ to -0.002$)$ & $<0.0001$ & $-0.007(-0.008$ to -0.005$)$ & $<0.0001$ \\
\hline Model 2 & $-0.003(-0.004$ to -0.002$)$ & $<0.0001$ & $-0.007(-0.008$ to -0.005$)$ & $<0.0001$ \\
\hline Model 3 & $0.000(-0.001$ to 0.002$)$ & Ns & $-0.005(-0.007$ to -0.003$)$ & $<0.0001$ \\
\hline \multicolumn{5}{|l|}{ Lumbar spine } \\
\hline Unadjusted & $-0.003(-0.005$ to -0.001$)$ & 0.003 & $-0.007(-0.009$ to -0.005$)$ & $<0.0001$ \\
\hline Model 1 & $-0.001(-0.002$ to 0.001$)$ & Ns & $-0.003(-0.005$ to -0.002$)$ & $<0.0001$ \\
\hline Model 2 & $-0.001(-0.002$ to 0.001$)$ & Ns & $-0.003(-0.005$ to -0.002$)$ & $<0.0001$ \\
\hline Model 3 & $0.000(-0.001$ to 0.002$)$ & Ns & $-0.003(-0.006$ to -0.001$)$ & 0.01 \\
\hline
\end{tabular}

Model 1 adjusted for age, body mass index (BMI), presence of prevalent fragility fractures, family history of osteoporosis, and menopause. Model 2 adjusted for age, body mass index (BMI), presence of prevalent fragility fractures, family history of osteoporosis, menopause, glucocorticoid treatment, and comorbidities. Model 3 (main model) adjusted for age, body mass index (BMI), presence of prevalent fragility fractures, family history of osteoporosis, menopause, glucocorticoid treatment, comorbidities, and macro-area of residency (categorized as northern Italy, central Italy, and southern Italy)

study. However, several aspects differentiate our analyses from the study by Ranzani et al. First, the authors involved approximately 4,000 individuals in their study compared to slightly less than 60,000 patients included in our study. Moreover, Ranzani et al included younger individuals (mean age 35.7 years), who were exposed to an average exposure to PM2.5 of $32.8 \mu \mathrm{g} / \mathrm{m}^{3}$, which is remarkably higher than the average PM2.5 concentration observed in Italy $\left(16.0 \mu \mathrm{g} / \mathrm{m}^{3}\right)$. Nevertheless, other population-based studies had been conducted on the effects of air pollution on bone health. For example, Chang and colleagues found an association between exposure to PM and osteoporosis in a cohort of more than 36,000 Taiwanese residents [21]. However, in their study, the diagnosis of osteoporosis was based on administrative data and was not supported by densitometric data, limiting the possibility of analyzing BMD as a continuous variable as we did. Indeed, we retrieved patients' data from the DeFRAcalc79 database. The DeFRAcalc79 is a web-based fracture risk assessment tool that is commonly accessed for fracture risk assessment. The present study and other previously published studies showed the potentiality of datasets derived from such tools $[11,12,22]$.

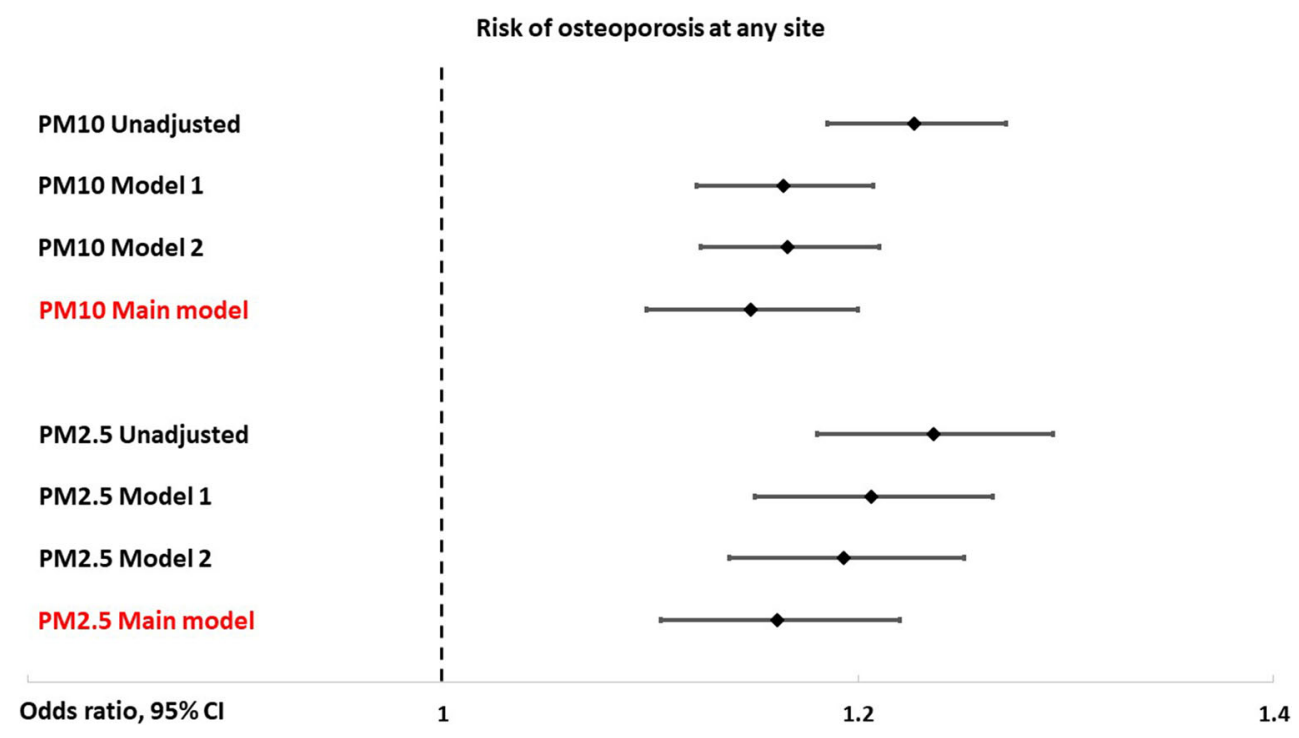

Fig. 2 Risk of osteoporosis at any site in patients chronically exposed to particulate matter (PM) $10>30 \mu \mathrm{g} / \mathrm{m}^{3}$ and PM2.5 $>25 \mu \mathrm{g} / \mathrm{m}^{3}$. Model 1 adjusted for age, body mass index (BMI), presence of prevalent fragility fractures, family history of osteoporosis, and menopause. Model 2 adjusted for age, body mass index (BMI), presence of prevalent fragility fractures, family history of osteoporosis, menopause, glucocorticoid

treatment, and comorbidities. Model 3 (main model) adjusted for age, body mass index (BMI), presence of prevalent fragility fractures, family history of osteoporosis, menopause, glucocorticoid treatment, comorbidities, and macro-area of residency (categorized as northern Italy, central Italy, and southern Italy) 


\section{PM10 Unadjusted \\ PM10 Model 1 \\ PM10 Model 2 \\ PM10 Main model \\ PM2.5 Unadjusted \\ PM2.5 Model 1 \\ PM2.5 Model 2 \\ PM2.5 Main model}

Risk of osteoporosis at femoral neck

1
1
1
1
1
1
1
1
1
1
1
1
1
1
1
1
1

Odds ratio, $95 \% \mathrm{Cl}$

1

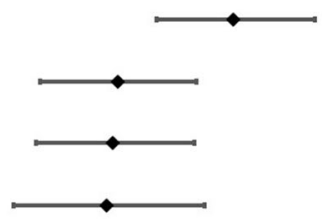

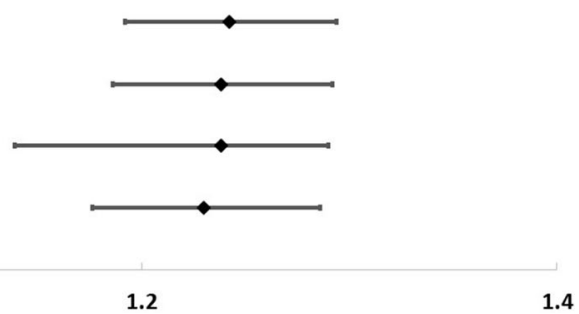

Fig. 3 Risk of osteoporosis at femoral neck in patients chronically exposed to particulate matter (PM) $10>30 \mu \mathrm{g} / \mathrm{m}^{3}$ and PM2.5 $>25 \mu \mathrm{g} /$ $\mathrm{m}^{3}$. Model 1 adjusted for age, body mass index (BMI), presence of prevalent fragility fractures, family history of osteoporosis, and menopause. Model 2 adjusted for age, body mass index (BMI), presence of prevalent fragility fractures, family history of osteoporosis, menopause,

Our study adds to the body of literature on this topic and provides new insight on the magnitude of the detrimental effect of PM exposure on bone health. Our study, different from others on the same topic, has been conducted on a large cohort of women at high risk of fracture with access to densitometric and bone-related clinical variables. However, the present study should be interpreted in view of some limitations. We

glucocorticoid treatment, and comorbidities. Model 3 (main model) adjusted for age, body mass index (BMI), presence of prevalent fragility fractures, family history of osteoporosis, menopause, glucocorticoid treatment, comorbidities, and macro-area of residency (categorized as northern Italy, central Italy, and southern Italy)

estimated the long-term exposure to air pollutant from the average concentration of PM in the time frame 2013 to 2019. Such estimation might not truly representative of the mean exposure during the lifespan of every patient of our study but rather a mere approximation of the long-term exposure. However, the vast majority of the studies that evaluated the effects of pollutants on health-related outcomes used

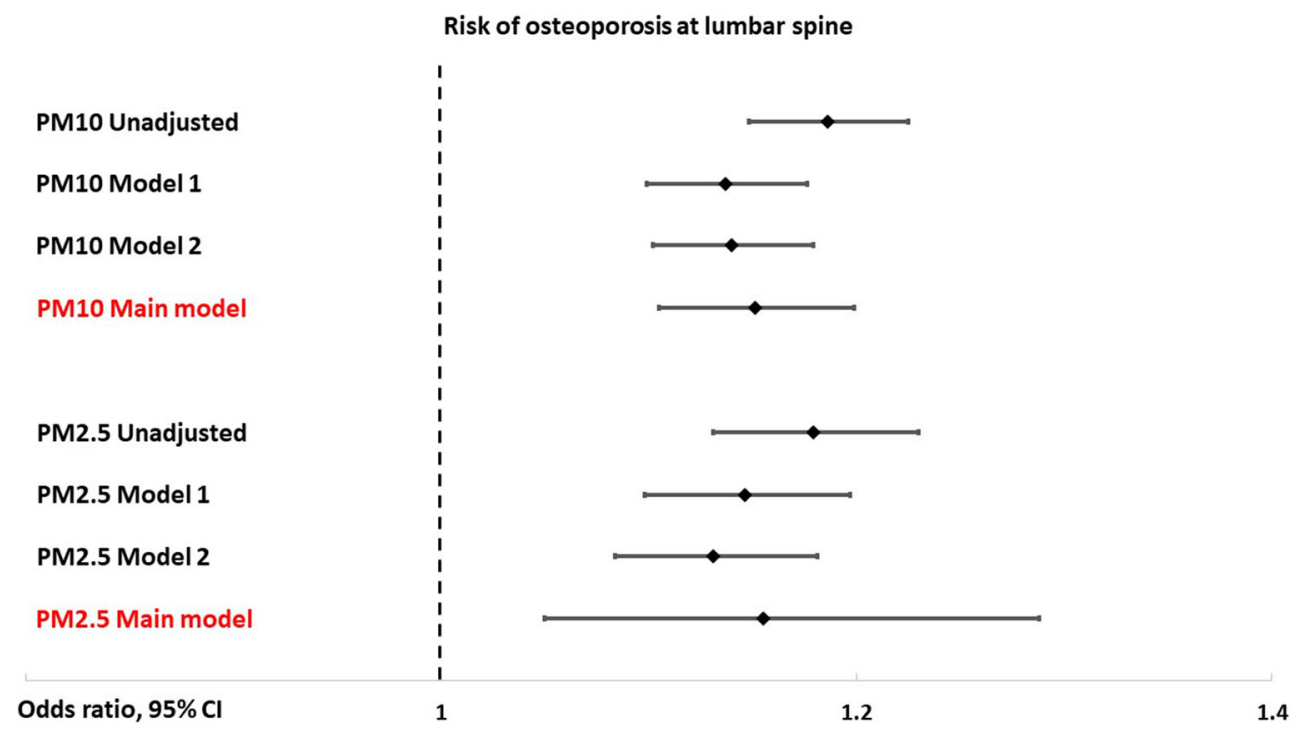

Fig. 4 Risk of osteoporosis at lumbar spine in patients chronically exposed to particulate matter (PM) $10>30 \mu \mathrm{g} / \mathrm{m}^{3}$ and PM2.5 $>25 \mu \mathrm{g} /$ $\mathrm{m}^{3}$. Model 1 adjusted for age, body mass index (BMI), presence of prevalent fragility fractures, family history of osteoporosis, and menopause. Model 2 adjusted for age, body mass index (BMI), presence of prevalent fragility fractures, family history of osteoporosis, menopause, glucocorticoid treatment, and comorbidities. Model 3 (main model) adjusted for age, body mass index (BMI), presence of prevalent fragility fractures, family history of osteoporosis, menopause, glucocorticoid treatment, comorbidities, and macro-area of residency (categorized as northern Italy, central Italy, and southern Italy) 
similar rough estimations of long-term exposure $[8,15]$. We did not have access to relocation data of the cohort. However, the internal mobility rate of the Italian population attests at approximately $2.2 \%$ of the population, and this proportion drops to less than $1 \%$ in individuals aged more than 50 years (https://www.istat.it/it/files/2018/12/Report-MigrazioniAnno-2017.pdf). In addition, it is reasonably to think that people living with osteoporosis or other diseases are unlikely to relocate at these rates. Thus, we can conservatively assume that less than 600 subjects in our cohort have relocated every year, a proportion that is unlikely to affect our results. Moreover, other pollutants (e.g., nitric oxide, sulfur dioxide, and carbon monoxide), which might play an important role in the development of osteoporosis, were not included in the study. We did not have access to data regarding scholarity or socioeconomic status, conditioning osteoporosis awareness, or physical activity and calcium intake, well-known risk factors for osteoporosis. Moreover, we did not have access to other covariates that might work has proxies of the socioeconomic status, such as income. Finally, our study has been conducted on a cohort of older women with higher risk for osteoporosis, which has affected the generalizability of our results.

In conclusion, we observed that exposure to airborne fine PM increased the risk of osteoporosis. Cortical bone seemed to be more susceptible compared to trabecular bone. Our study has important direct societal and clinical consequences: policies aimed at reducing particles emissions and their gaseous precursors would be relevant also in order to prevent osteoporosis; this recommendation is of particular interest in those highly industrialized regions where emissions are rapidly surging, and population is aging faster than ever.

Patient and public involvement statement This research was done without patient involvement. Patients were not invited to comment on the study design and were not consulted to develop patient relevant outcomes or interpret the results. Patients were not invited to contribute to the writing or editing of this document for readability or accuracy.

Data sharing No additional data available.

Transparency declaration The lead author (the manuscript's guarantor) affirms that the manuscript is an honest, accurate, and transparent account of the study being reported; that no important aspects of the study have been omitted; and that any discrepancies from the study as planned (and, if relevant, registered) have been explained.

Funding Open access funding provided by Università degli Studi di Verona within the CRUI-CARE Agreement.

\section{Declarations}

Ethics approval The study was conducted according to the protocol DEFRA 1876CESC approved by the Ethics Committee of the University of Verona Hospital, in accordance with the 1964 Helsinki declaration and its later amendments or comparable ethical standards.

Conflicts of interest All authors have completed the ICMJE uniform disclosure form at www.icmje.org/coi_disclosure.pdf (available on request from the corresponding author) and declare no conflicts of interest.

Open Access This article is licensed under a Creative Commons Attribution-NonCommercial 4.0 International License, which permits any non-commercial use, sharing, adaptation, distribution and reproduction in any medium or format, as long as you give appropriate credit to the original author(s) and the source, provide a link to the Creative Commons licence, and indicate if changes were made. The images or other third party material in this article are included in the article's Creative Commons licence, unless indicated otherwise in a credit line to the material. If material is not included in the article's Creative Commons licence and your intended use is not permitted by statutory regulation or exceeds the permitted use, you will need to obtain permission directly from the copyright holder. To view a copy of this licence, visit http:// creativecommons.org/licenses/by-nc/4.0/.

\section{References}

1. Black DM, Rosen CJ (2016) Clinical practice. Postmenopausal osteoporosis. N Engl J Med 374:254-262. https://doi.org/10. 1056/NEJMcp1513724

2. Burge R, Dawson-Hughes B, Solomon DH, Wong JB, King A, Tosteson A (2007) Incidence and economic burden of osteoporosis-related fractures in the United States, 2005-2025. J Bone Miner Res 22:465-475. https://doi.org/10.1359/jbmr.061113

3. Adami G, Saag KG (2019) Glucocorticoid-induced osteoporosis: 2019 concise clinical review. Osteoporos Int 30:1145-1156. https:// doi.org/10.1007/s00198-019-04906-x

4. Adami G, Saag KG (2019) Osteoporosis pathophysiology, epidemiology, and screening in rheumatoid arthritis. Curr Rheumatol Rep 21:34. https://doi.org/10.1007/s11926-019-0836-7

5. Adami G, Fassio A, Rossini M, Caimmi C, Giollo A, Orsolini G, Viapiana O, Gatti D (2019) Osteoporosis in rheumatic diseases. Int J Mol Sci 20. https://doi.org/10.3390/ijms20235867

6. Nguyen TV (2017) Air pollution: a largely neglected risk factor for osteoporosis. Lancet Planet Health 1:e311-e312. https://doi.org/10. 1016/S2542-5196(17)30143-2

7. Saha H, Mukherjee B, Bindhani B, Ray MR (2016) Changes in RANKL and osteoprotegerin expression after chronic exposure to indoor air pollution as a result of cooking with biomass fuel. J Appl Toxicol 36:969-976. https://doi.org/10.1002/jat.3275

8. Prada D, López G, Solleiro-Villavicencio H, Garcia-Cuellar C, Baccarelli AA (2020) Molecular and cellular mechanisms linking air pollution and bone damage. Environ Res 185:109465. https:// doi.org/10.1016/j.envres.2020.109465

9. Arias-Pérez RD, Taborda NA, Gómez DM, Narvaez JF, Porras J, Hernandez JC (2020) Inflammatory effects of particulate matter air pollution. Environ Sci Pollut Res Int 27:42390-42404. https://doi. org/10.1007/s11356-020-10574-w

10. Mousavi SE, Amini H, Heydarpour P, Amini Chermahini F, Godderis L (2019) Air pollution, environmental chemicals, and 
smoking may trigger vitamin D deficiency: evidence and potential mechanisms. Environ Int 122:67-90. https://doi.org/10.1016/j. envint.2018.11.052

11. Adami G, Gatti D, Rossini M, Orsolini G, Pollastri F, Bertoldo E, Viapiana O, Bertoldo F, Giollo A, Fassio A (2020) Risk of fragility fractures in obesity and diabetes: a retrospective analysis on a nation-wide cohort. Osteoporos Int 31:2113-2122. https://doi.org/ 10.1007/s00198-020-05519-5

12. Adami G, Giollo A, Rossini M, Orsolini G, Benini C, Viapiana O, Gatti D, Fassio A (2020) Different fracture risk profile in patients treated with anti-osteoporotic drugs in real-life. Reumatismo 72: 71-74. https://doi.org/10.4081/reumatismo.2020.1267

13. Almeida M, O'Brien CA (2013) Basic biology of skeletal aging: role of stress response pathways. J Gerontol A Biol Sci Med Sci 68: 1197-1208. https://doi.org/10.1093/gerona/glt079

14. Almeida M, Ambrogini E, Han L, Manolagas SC, Jilka RL (2009) Increased lipid oxidation causes oxidative stress, increased peroxisome proliferator-activated receptor-gamma expression, and diminished pro-osteogenic Wnt signaling in the skeleton. J Biol Chem 284:27438-27448. https://doi.org/10.1074/jbc.M109.023572

15. Prada D, Zhong J, Colicino E, Zanobetti A, Schwartz J, Dagincourt N, Fang SC, Kloog I, Zmuda JM, Holick M, Herrera LA, Hou L, Dominici F, Bartali B, Baccarelli AA (2017) Association of air particulate pollution with bone loss over time and bone fracture risk: analysis of data from two independent studies. Lancet Planet Health 1:e337-e347. https://doi.org/10.1016/S2542-5196(17) 30136-5

16. Ritchlin CT, Haas-Smith SA, Li P, Hicks DG, Schwarz EM (2003) Mechanisms of TNF- $\alpha$ - and RANKL-mediated osteoclastogenesis and bone resorption in psoriatic arthritis. J Clin Investig 111:821831. https://doi.org/10.1172/JCI16069
17. Liu Q, Gu X, Deng F, Mu L, Baccarelli AA, Guo X, Wu S (2019) Ambient particulate air pollution and circulating $\mathrm{C}$-reactive protein level: a systematic review and meta-analysis. Int J Hyg Environ Health 222:756-764. https://doi.org/10.1016/j.ijheh.2019.05.005

18. Adami G, Viapiana O, Rossini M, Orsolini G, Bertoldo E, Giollo A, Gatti D, Fassio A (2021) Association between environmental air pollution and rheumatoid arthritis flares. Rheumatology (Oxford). https://doi.org/10.1093/rheumatology/keab049

19. Franceschi C, Garagnani P, Parini P, Giuliani C, Santoro A (2018) Inflammaging: a new immune-metabolic viewpoint for age-related diseases. Nat Rev Endocrinol 14:576-590. https://doi.org/10.1038/ s41574-018-0059-4

20. Ranzani OT, Milà C, Kulkarni B, Kinra S, Tonne C (2020) Association of ambient and household air pollution with bone mineral content among adults in peri-urban South India. JAMA Netw Open 3:e1918504. https://doi.org/10.1001/jamanetworkopen. 2019.18504

21. Chang K-H, Chang M-Y, Muo C-H, Wu TN, Hwang BF, Chen CY, Lin TH, Kao CH (2015) Exposure to air pollution increases the risk of osteoporosis: a nationwide longitudinal study. Medicine (Baltimore) 94:e733. https://doi.org/10.1097/MD. 0000000000000733

22. Adami G, Gatti D, Rossini M, Giollo A, Bertoldo E, Viapiana O, Olivi P, Fassio A (2021) Factors associated with referral for osteoporosis care in men: a real-life study of a nationwide dataset. Arch Osteoporos 16:56. https://doi.org/10.1007/s11657-021-00915-8

Publisher's note Springer Nature remains neutral with regard to jurisdictional claims in published maps and institutional affiliations. 